\title{
Effective cultivation of microalgae for biofuel production: a pilot-scale evaluation of a novel oleaginous microalga Graesiella sp. WBG-1
}

Xiaobin Wen ${ }^{1}$, Kui Du ${ }^{1,2}$, Zhongjie Wang ${ }^{1}$, Xinan Peng ${ }^{1,2}$, Liming Luo ${ }^{1,3}$, Huanping Tao ${ }^{1}$, Yan Xu ${ }^{1,2}$, Dan Zhang ${ }^{1}$, Yahong Geng ${ }^{1}$ and Yeguang Li $^{1^{*}}$

\begin{abstract}
Background: Commercial production of microalgal biodiesel is not yet economically viable, largely because of low storage lipid yield in microalgae mass cultivation. Selection of lipid-rich microalgae, thus, becomes one of the key research topics for microalgal biodiesel production. However, the laboratory screening protocols alone cannot predict the ability of the strains to dominate and perform in outdoor ponds. Comprehensive assessment of microalgae species should be performed not only under the laboratory conditions, but also in the fields.

Results: Laboratory investigations using a bubbled column photobioreactor indicated the microalga Graesiella sp. WBG-1 to be the most productive species among the 63 Chlorophyta strains. In a $10 \mathrm{~L}$ reactor, mimicking the

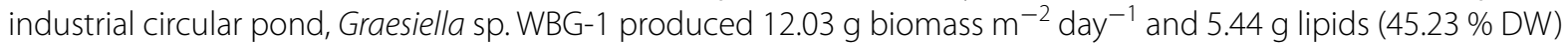
$\mathrm{m}^{-2}$ day $^{-1}$ under $15 \mathrm{~mol} \mathrm{~m}^{-2}$ day $^{-1}$ artificial light irradiations. The lipid content decreased to $~ 34 \%$ DW when the microalga was cultured in $30 \mathrm{~L}$ tank PBR under natural solar irradiations, but the decline of lipid content with scaling up was the minimum among the tested strains. Based on these results, the microalga was further tested for its lipid production and culture competitiveness using a pilot-scale raceway pond $\left(200 \mathrm{~m}^{2}\right.$ illuminated area, culture volume 40,000 L). Consequently, Graesiella sp. WBG-1 maintained a high lipid content (33.4 \% DW), of which 90 \% was storage TAGs. Results from the outdoor experiments indicated the nice adaptability of the Graesiella sp. WBG-1 to strong and fluctuating natural solar irradiance and temperature, and also demonstrated several other features, such as large cell size (easy for harvest and resistant to swallow by protozoa) and tolerance to high culture pH (helpful to $\mathrm{CO}_{2}$ fixation).

Conclusions: Graesiella sp. WBG-1 was a promising strain capable of accumulating large amount of storage lipid under nature solar irradiance and temperature. The high lipid content of $33.4 \%$ DW was achieved for the first time in pilot-scale raceway pond. The results also provide evidence for the feasibility of using low-cost raceway pond for autotrophic cultivation of microalgae for biodiesel production.
\end{abstract}

Keywords: Graesiella, Microalgae selection, Lipid, Mass culture, Open raceway pond

\footnotetext{
*Correspondence: yeguang@wbgcas.cn

${ }^{1}$ Key Laboratory of Pant Germplasm Enhancement and Specialty

Agriculture, Wuhan Botanical Garden, Chinese Academy of Sciences,

Wuhan 430074, China

Full list of author information is available at the end of the article
} 


\section{Background}

In recent years, mass production of microalgae has been extensively exploited for applications in the industry of food, aquaculture, and bioenergy, and especially for biodiesel production $[1,2]$. However, there is still a large margin to improve economic yield for large-scale biofuel production [3, 4], since fossil fuel is still much cheaper and serves as the dominant energy source nowadays.

One of the obstacles making microalgal biodiesel commercially unfeasible is the low yield of storage lipid in outdoor microalgae mass cultivation $[3,5]$. So far, the algal biomass productivities achieved in large-scale raceway pond do not exceed 20-40 g dry weight $\mathrm{m}^{-2}$ day $^{-1}$, and a maximum solar-to-biomass conversion efficiency of $3 \%$ was reported $[6,7]$. Furthermore, high lipid accumulation is more difficult to achieve. Although Chlorella, Scenedesmus, Neochloris, and Nanochloropsis strains are repeatedly reported to give an average lipid content of 40-60\% in dry cell mass in laboratory [8, 9], outdoor cultivation of these strains has only been accomplished on very small scale and a total lipid content of $30 \%$ has rarely been achieved [10-12]. The Aquatic Species Program (ASP) spent considerable effort in isolation, screening, genetic improvement, and outdoor cultivation of microalgae strains. At its peak, the collection of ASP contained over 3000 strains of lipid-rich microalgae [13]. However, there is still lack of robust strains capable of high lipid productivity in outdoor large-scale cultivation $[6,13]$. In its close-out report, ASP pointed out that the laboratory-level screening protocols have relatively little predictive power for the ability of the strains to dominate and perform in outdoor ponds [13].

To fill the gap between laboratory experiment and field test, future research should cover the whole chain of process development in an integrated and iterative way [6]. In this context, selection and assessment of robust microalgal strains for large-scale cultivation have become one of the key research topics for biodiesel production. Small-scale open systems should be used as selection devices for microalgae strains suitable for outdoor mass culture [13]. Full-scale assessment of lipid-rich microalgae should be performed not only under the laboratory conditions, but also in the fields, to test their adaptability to changes in temperature, strong light irradiance, and other chemical and biological environment conditions.

Graesiella sp. WBG-1 is a unicellular green microalga with broadly ellipsoidal or globose cells. This strain was originally isolated from Chenghai Lake, Yunnan province, China, by the researchers in our lab. Molecular analysis contributed the WBG-1 strain mainly to genus Graesiella and showed $99.8 \%$ similarity with two Graesiella species: Graesiella emersonii and Graesiella vacuolata based on the $18 \mathrm{~S}$ rDNA/ITS sequence. Preliminary investigations using a bubbled column photobioreactor showed Graesiella sp. WBG-1 to be one of the most productive microalgae among the 63 studied Chlorophyta strains (Additional file 1). Furthermore, this strain also possesses some other desirable features, such as large cell size (easy to harvest) and high adaptive capacity to a wide range of culture $\mathrm{pH}$. All the above advantages together encouraged us to perform a comprehensive selection and assessment using this promising strain Graesiella sp. WBG-1.

Given the above considerations, we carried out both indoor and outdoor experiments to study the effects of several fundamental factors on the growth and lipid accumulation of the microalga Graesiella sp. WBG-1, and to test its robustness under the outdoor environmental conditions in a $200 \mathrm{~m}^{2}$ raceway pond. $\mathrm{CO}_{2}$ utilization and other features of Graesiella sp. WBG-1 were also discussed.

\section{Methods}

\section{Growth medium and preparation}

The basal growth medium for microalgae culture was a modified BG-11 medium which had the following components (per liter): $\mathrm{NaNO}_{3}(100 \mathrm{mg}), \mathrm{K}_{2} \mathrm{HPO}_{4} \cdot 3 \mathrm{H}_{2} \mathrm{O}$ (40 mg), $\mathrm{MgSO}_{4} \cdot 7 \mathrm{H}_{2} \mathrm{O}(75 \mathrm{mg}), \mathrm{CaCl}_{2} \cdot 2 \mathrm{H}_{2} \mathrm{O}(36 \mathrm{mg})$, citric acid $(6 \mathrm{mg})$, Fe-ammonium citrate (6 mg), EDTA $\cdot \mathrm{Na}_{2}$ (1 mg), $\mathrm{NaHCO}_{3}(20 \mathrm{mg}), \mathrm{H}_{3} \mathrm{BO}_{3}(2.86 \mathrm{mg}), \mathrm{MnCl}_{2} \cdot 4 \mathrm{H}_{2} \mathrm{O}$ (1.8 mg), $\mathrm{ZnSO}_{4} \cdot 7 \mathrm{H}_{2} \mathrm{O}(0.22 \mathrm{mg}), \mathrm{CuSO}_{4} \cdot 5 \mathrm{H}_{2} \mathrm{O}(0.08 \mathrm{mg})$, $\mathrm{Na}_{2} \mathrm{MoO}_{4} \cdot 2 \mathrm{H}_{2} \mathrm{O} \quad(0.391 \mathrm{mg})$, and $\mathrm{Co}\left(\mathrm{NO}_{3}\right)_{2} \cdot 6 \mathrm{H}_{2} \mathrm{O}$ $(0.0494 \mathrm{mg})$. Sodium nitrate and sodium bicarbonate concentrations were modified as indicated in the text.

The BG-11 medium was prepared with deionized water for laboratory experiments, and with water from Chenghai Lake (containing $\sim 1 \mathrm{~g} \mathrm{~L}^{-1} \mathrm{NaHCO}_{3}$ ) for outdoor experiments. The deionized water was sterilized by autoclave, and the water from Chenghai Lake was purified by filtration, and then, the sterile nutrient stock solutions were added to either the deionized water or the water from Chenghai Lake to make final medium. The culture pond was exposed to direct solar irradiation for at least $12 \mathrm{~h}$ before medium preparation.

\section{Bubbled column PBR cultivation}

Each column of the bubbled column PBR used in this study has an inner diameter of $3 \mathrm{~cm}$ and a working volume of $200 \mathrm{~mL}$. The columns were illuminated with white fluorescent tubes for $14 \mathrm{~h}$ every day, and the light intensity at the reactor surface was $300 \mu \mathrm{mol} \mathrm{m}{ }^{-2} \mathrm{~s}^{-1}$ $\left(15 \mathrm{~mol}\right.$ photons $\left.\mathrm{m}^{-2} \mathrm{day}^{-1}\right) . \mathrm{CO}_{2}$-enriched $(1 \%, \mathrm{v} / \mathrm{v})$ air was injected at the bottom of the column through a glass tube, that is used for $\mathrm{CO}_{2}$ supplement, culture mixing, and $\mathrm{O}_{2}$ exchange. The air flow rate was maintained at $250 \mathrm{~mL} \mathrm{~min}{ }^{-1}$. A thermostatic water circulator was used 
as water bath to keep the temperature of the culture columns at $30^{\circ} \mathrm{C}$.

The algal cells were harvested from seed culture and resuspended into sterilized BG-11 medium for inoculation. The cells were grown in batch with an initial cell density of $0.5 \pm 0.05$ (optical density at $540 \mathrm{~nm}$ ). To investigate the effects of nitrate and bicarbonate concentration on cell growth and lipid accumulation, different doses of sodium nitrate or sodium bicarbonate were added into each column before inoculation. All of the experiments were carried out in triplicate.

\section{Scale-up cultivation in a $10 \mathrm{~L}$ circular pond}

A mini circular pond cultivation system was used to simulate the outdoor open pond cultivation under artificial illumination. The circular pond was equipped with four units to control mixing, irradiance, temperature, and $\mathrm{pH}$, as described in previous study [14].

The cells were grown in batch with an initial cell density of $0.1 \pm 0.05$ (optical density at $540 \mathrm{~nm}$ ). Total culture volume was about $10 \mathrm{~L}$ giving a culture depth (light path) of $10 \mathrm{~cm}$, and the effective illuminated area was $0.1 \mathrm{~m}^{2}$. Banks of white fluorescent tubes were placed above the pond, and provided $300 \mu \mathrm{mol}$ photons $\mathrm{m}^{-2} \mathrm{~s}^{-1}$ with $14 \mathrm{~h}: 10 \mathrm{~h}$ light-dark cycle $\left(15 \mathrm{~mol}\right.$ photons $\mathrm{m}^{-2}$ day $\left.{ }^{-1}\right)$. The cultures were kept at $30^{\circ} \mathrm{C}$ and mixed at $50 \mathrm{r} \mathrm{min}^{-1}$ continuously. Pure $\mathrm{CO}_{2}$ was dispersed into the culture suspension through a gas diffuser under the programming control of an online $\mathrm{pH}$ sensor, to maintain the culture $\mathrm{pH}$ within a desired range. In another study, we found that Graesiella sp. WBG-1 was well adapted to culture $\mathrm{pH}$ range of 7-10 (unpublished data). Therefore, the culture $\mathrm{pH}$ of $9.0 \pm 0.5$ was maintained in the simulate experiments to facilitate external $\mathrm{CO}_{2}$ uptake. The experiments were carried out in duplicate.

\section{Scale-up cultivation in a $30 \mathrm{~L}$ tank PBR}

To test the adaptability of the strain to variable environmental conditions, outdoor scale-up cultivations of the Graesiella sp. WBG-1 were carried out in $30 \mathrm{~L}$ tank PBR under natural light illumination.

The PBR used in this experiment consisted of four open-top polyethylene tanks placed side-by-side in a single row on a concrete platform. The working volume is $30 \mathrm{~L}$ for each culture tank, giving an illuminated area of $0.19 \mathrm{~m}^{2}$. An electromagnetic air compressor continuously blew sterilized air into the culture suspension during cultivation. The air flow was maintained at $3 \mathrm{~L} \mathrm{~min}^{-1}$. The air was enriched with $\mathrm{CO}_{2}(1 \%, \mathrm{v} / \mathrm{v})$ during light period to support cell growth and maintain $\mathrm{pH}$ within the desired range $(9.0 \pm 0.5)$. Natural solar irradiance and environmental temperature were monitored and logged on site with an automatic weather station.

\section{Outdoor cultivation in $200 \mathrm{~m}^{2}$ raceway pond}

Pilot-scale evaluation of Graesiella sp. WBG-1 was carried out at Chenghai Lake, Yunnan province, China, $\left(\mathrm{N} 26^{\circ} 29^{\prime} 29.64^{\prime \prime} \mathrm{E} 100^{\circ} 40^{\prime} 56.12^{\prime \prime}\right)$ using a traditional raceway pond.

The raceway pond was an open system constructed of concrete blocks. The pond was $20 \mathrm{~m}$ long, $12 \mathrm{~m}$ wide, giving an effective culture area of $200 \mathrm{~m}^{2}$. Two large paddle wheels were installed apart within the raceway for mixing, and were driven by speed-adjustable motors and able to provide a flow velocity of $10-60 \mathrm{~cm} \mathrm{~s}^{-1}$. The liquid flow velocity was set at $45 \mathrm{~cm} \mathrm{~s}^{-1}$ in this study, and the paddle wheels were turned on in daytime and turned off in night. Pure $\mathrm{CO}_{2}$ was automatically injected into the culture via a $3 \mathrm{~m}$-long microporous polymer tube (gas diffuser) that was placed at the bottom of the culture pond. The automatic injection of $\mathrm{CO}_{2}$, which was controlled by an online $\mathrm{pH}$ sensor, maintained the culture $\mathrm{pH}$ within the desired range $(\mathrm{pH} 9.0 \pm 0.5)$. The working culture depth (light path) was $20 \mathrm{~cm}$, corresponding to 40,000 L culture volume. Natural solar irradiance, environmental temperature, and suspension temperature were monitored and logged on site with an automatic weather station.

Two similar but smaller raceway ponds $\left(20 \mathrm{~m}^{2}\right)$ covered by greenhouse were used for high-quality seed culture preparation. Independent batch cultures in the $200 \mathrm{~m}^{2}$ pond were carried out three times in June 2013, July 2013, and May 2014.

\section{Analytical procedure}

Culture growth was estimated by measuring the dry biomass concentration of the culture broth. About $10 \mathrm{~mL}$ culture broth was filtered through a pre-dried GF/C glass microfiber filter paper (pore size $0.45 \mu \mathrm{m}$ ), and dried at $105^{\circ} \mathrm{C}$ for $4 \mathrm{~h}$, and then weighed to calculate dry biomass concentration (DW, in grams of biomass per liter of culture broth). Daily biomass productivity was calculated by dividing the difference between the DWs at the start time and the end time by its duration (days). The microalga was stained with Nile Red according to Li's method [10] and observed using a Nikon Eclipse 80i microscope.

A spectrophotometric method, described by Collos et al. [15], was used to monitor residual nitrate concentration. Briefly, the absorbance of culture filtrate $(0.22 \mu \mathrm{m}$ filter) at $220 \mathrm{~nm}$ and a pre-constructed standard curve were used to determine residual nitrate.

Cells were collected by centrifugation $(5000 \mathrm{rpm}$ for $5 \mathrm{~min})$ and lyophilized $\left(-56{ }^{\circ} \mathrm{C}\right.$ cryotrapping, $10-14 \mathrm{~Pa}$ vacuum) for biochemical analysis. For lipid quantification, $50 \mathrm{mg}$ of dry algal biomass was fully grounded, transferred to a covered centrifuge tube, and then extracted with a mixture of $n$-hexane and ethyl acetate 
$(1: 1, \mathrm{v}: \mathrm{v})$ for $20 \mathrm{~min}$. The extraction was repeated three times, and all extracts were combined into a pre-weighed glass tube, and then dried under nitrogen protection. The lipids were determined gravimetrically.

Neutral lipid (Triacylglycerides, TAGs) was fractionated from the lipid extracts by column chromatography using a $2 \mathrm{~cm} \times 20 \mathrm{~cm}$ column packed with 4 g silica gel 60 [16]. The lipid extracts $(\sim 100 \mathrm{mg})$ were dissolved in $2 \mathrm{~mL}$ chloroform and loaded onto the column. TAGs were eluted from the column by $20 \mathrm{~mL}$ chloroform. The eluted TAGs fraction were confirmed by TLC, and then dried and quantified gravimetrically.

To analyze fatty acids, about $20 \mathrm{mg}$ of the lipid extract was dissolved in $3 \mathrm{~mL} n$-hexane and then transmethylated by adding $3 \mathrm{~mL}$ methanol- $\mathrm{KOH}(0.5 \% \mathrm{KOH})$ and heating at $50{ }^{\circ} \mathrm{C}$ for $60 \mathrm{~min}$. After cooling to room temperature, the hexane layer was separated and dried with anhydrous sodium sulfate. Fatty acid methyl esters were analyzed by gas chromatography (Agilent 7890A) using an HP-5 Phenyl Methyl Siloxan column $(30 \mathrm{~m} \times 0.32 \mathrm{~mm} \times 0.25 \mu \mathrm{m})$ and a flame ionization detector. $1 \mu \mathrm{L}$ fatty acid methyl esters solution was injected to the sampler with a splitting ratio of 5:1. The heating program was $150{ }^{\circ} \mathrm{C}$ held for $2 \mathrm{~min}$, then increased to $250{ }^{\circ} \mathrm{C}$ at a rate of $10{ }^{\circ} \mathrm{C}$ per min, and held for $8 \mathrm{~min}$. A standard FAME Mix (Sigma-Aldrich) was used for fatty acid identification.

About $10 \mathrm{mg}$ of lyophilized algal powder was used for total carbohydrate quantification. The algal powder was transferred to a covered tube, fully mixed with $1 \mathrm{~mL}$ hydrochloric acid $(6 \mathrm{M})$, to digest at $105^{\circ} \mathrm{C}$ for $1 \mathrm{~h}$. After cooling to RT, about $1 \mathrm{~mL} \mathrm{NaOH}(6 \mathrm{M})$ was added into the solution to neutralize acid, followed by centrifugation at $3500 \mathrm{~g}$ for $5 \mathrm{~min}$. The supernatant was collected into a new tube and its volume was brought to $2 \mathrm{~mL}$ with deionized $\mathrm{H}_{2} \mathrm{O} .100 \mu \mathrm{L}$ of the diluted supernatant was diluted again with deionized $\mathrm{H}_{2} \mathrm{O}$ to $2 \mathrm{~mL}$, mixed with $1 \mathrm{~mL}$ phenol (6\%), and then $5 \mathrm{~mL}$ sulfuric acid was trickled into the sample for color development. Finally, the optical density at $490 \mathrm{~nm}$ was measured on a spectrophotometer. To quantify total carbohydrate content, glucose was used to establish the standard curve.

Protein content was determined as described by Slocombe et al. [17]. Briefly, $10 \mathrm{mg}$ freeze-dried algal powder was suspended in $500 \mu \mathrm{L}$ of $24 \%(\mathrm{w} / \mathrm{v})$ trichloroacetic acid (TCA) and then incubated at $95{ }^{\circ} \mathrm{C}$ for $15 \mathrm{~min}$. The lysate containing $24 \%(\mathrm{w} / \mathrm{v})$ TCA were cooled to RT and diluted to $6 \%(\mathrm{w} / \mathrm{v})$ with $1.5 \mathrm{~mL}$ deionized water. The homogenate was centrifuged at $15,000 \mathrm{~g}$ for $20 \mathrm{~min}$ and the supernatant was discarded. The pellets were resuspended in $1 \mathrm{~mL} \mathrm{NaOH}(1 \mathrm{M})$ by repeated pipetting and then incubated at $40^{\circ} \mathrm{C}$ for $2 \mathrm{~h}$. The protein concentration was then spectrophotometrically measured according to standard Bradford assay.

\section{Calculations}

Areal biomass concentration $\left(C_{\text {biomass }}, \mathrm{g} \mathrm{m}^{-2}\right)$ was calculated by the following:

$$
C_{\text {biomass }}=\frac{\mathrm{DW} \times V}{S}
$$

with DW as measured biomass dry weight $\left(\mathrm{g} \mathrm{L}^{-1}\right), V$ as culture volume (L), and $S$ as illuminated area $\left(\mathrm{m}^{2}\right)$.

Biomass productivity $\left(P_{\text {biomass }}, \mathrm{g} \mathrm{m}^{-2}\right.$ day $\left.^{-1}\right)$ was calculated according to the following:

$$
P_{\text {biomass }}=\frac{C_{\text {biomass }, t 2}-C_{\text {biomass }, t 1}}{t 2-t 1}
$$

with $C_{\text {biomass, } t 2}$ and $C_{\text {biomass }, t 1}$ as biomass concentrations at culture time $t 2$ (day) and $t 1$ (day), respectively.

Lipid productivity $\left(P_{\text {lipid }}, \mathrm{g} \mathrm{m}^{-2}\right.$ day $\left.^{-1}\right)$ was calculated according to the following:

$$
P_{\text {lipid }}=\frac{C_{\text {biomass }, t 2} \times C_{\text {lipid }, t 2}-C_{\text {biomass }, t 1} \times C_{\text {lipid }, t 1}}{t 2-t 1}
$$

with $C_{\text {lipid, } t 2}$ and $C_{\text {lipid, } t 1}$ as lipid content at culture time $t 2$ (day) and $t 1$ (day), respectively.

Average daily light intensity $\left(I_{\mathrm{av}}, \mathrm{mol} \mathrm{m}^{-2}\right.$ day $\left.^{-1}\right)$ was calculated according to the following:

$$
I_{\mathrm{av}}=\frac{\sum_{0}^{t} I_{\mathrm{inc}}}{t}
$$

with $I_{\text {inc }}$ as daily incident light intensity $\left(\mathrm{mol} \mathrm{m}^{-2} \mathrm{day}^{-1}\right)$ measured by the automatic weather station.

Biomass-specific light availability ( $I_{\text {biomass }}$ mol g ${ }^{-1}$ day $^{-1}$ ) was calculated according to Ref. [18] by the following:

$$
I_{\text {biomass }}=\frac{I_{\mathrm{av}}}{C_{\text {biomass }}}
$$

$\mathrm{CO}_{2}$ utilization rate $\left(R_{\mathrm{c}}, \%\right)$ was calculated by the following:

$$
R_{\mathrm{c}}=\frac{W_{\mathrm{b}} \times 1000 \times 0.5 \div 12}{C_{1}-C_{2}+C_{3}} \times 100 \%
$$

with $W_{\mathrm{b}}$ as net increase of biomass $(\mathrm{kg})$ during cultivation; $C_{1}$ as dissolved inorganic carbon $\left(\left[\mathrm{H}_{2} \mathrm{CO}_{3}\right]+\left[\mathrm{CO}_{3}{ }^{2-}\right]+\left[\mathrm{HCO}_{3}{ }^{-}\right]\right.$, mol $)$in the medium at the begin of culture; $C_{2}$ as dissolved inorganic carbon (mol) in the medium at the end of culture; and $C_{3}$ as the total $\mathrm{CO}_{2}$ (mol) used during culture. The bio-fixated carbon ( $50 \%$ of the biomass is $C$ ) divided by consumed carbon was defined as $\mathrm{CO}_{2}$ utilization rate in this study. 


\section{Results}

Effects of nitrate and bicarbonate on cell growth of Graesiella sp. WBG-1 under the laboratory conditions

The microalga Graesiella sp. WBG-1 was cultivated in a $200 \mathrm{~mL}$ column photobioreactor under different bicarbonate and nitrate concentrations, to study the effects of carbon and nitrogen on the cell growth and lipid accumulation, which were essential for mass production optimization.

As shown in Fig. 1a, the biomass concentration $\left(C_{\text {biomass }}\right)$ of Graesiella sp. WBG-1 increased almost linearly with increasing concentrations of bicarbonate from 0 to $11.9 \mathrm{mM}$, and the increase was no more significant when the concentration of bicarbonate was above $11.9 \mathrm{mM}$. When the concentration of bicarbonate was increased to $47.62 \mathrm{mM}$, the $C_{\text {biomass }}$ of Graesiella sp. WBG-1 dropped significantly due to excessive salinity. In contrast, the lipid content $\left(C_{\text {lipid }}\right)$ of Graesiella sp. WBG-1 did not change under all tested bicarbonate concentrations (Fig. 1a).

The effects of nitrate on cell growth and lipid accumulation were shown in Fig. 1b. The $C_{\text {biomass }}$ of Graesiella
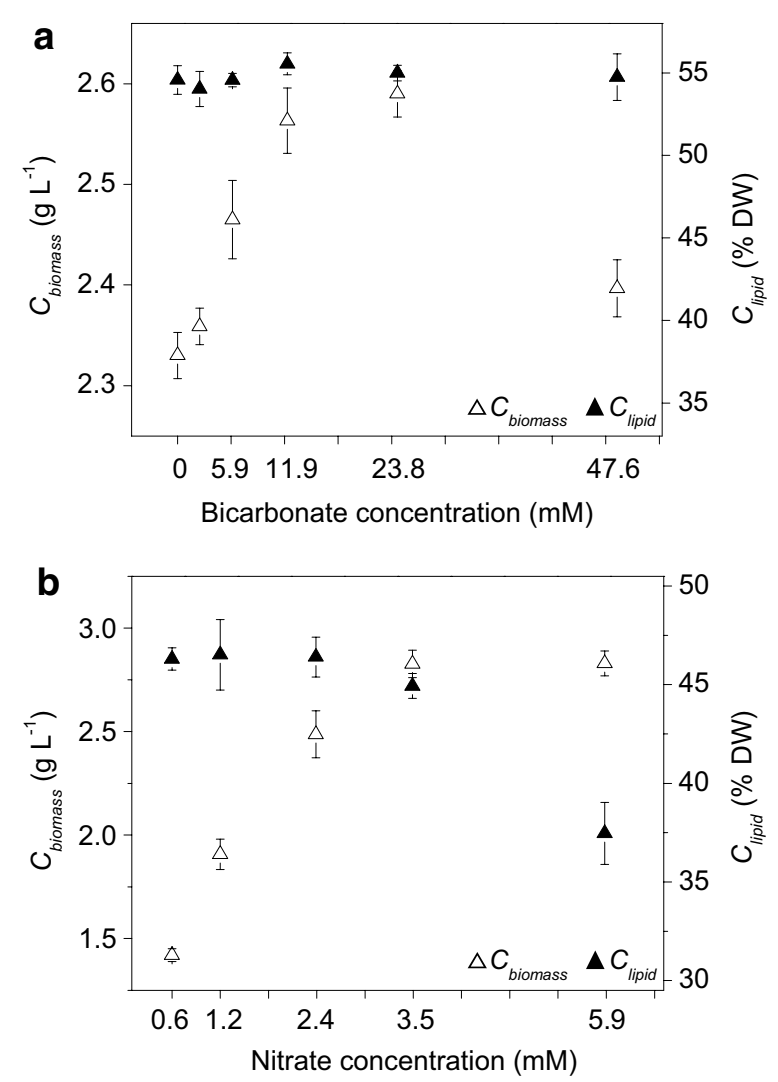

Fig. 1 Effects of bicarbonate (a) and nitrate (b) on cell growth (open triangle) and lipid accumulation (filled triangle) sp. WBG-1 increased with increasing nitrate concentrations, while the lipid content $\left(C_{\text {lipid }}\right)$ decreased gradually (Fig. 1b). The maximum $C_{\text {biomass }}$ of $\sim 2.8 \mathrm{~g} \mathrm{~L}^{-1}$ was achieved at $5.88 \mathrm{mM}$ nitrate, while the $C_{\text {lipid }}$ further decreased to $37.46 \% \mathrm{DW}$ at this concentration.

\section{Lipid production of Graesiella sp. WBG-1 in small-scale reactor}

As shown in Fig. 2a, in a $10 \mathrm{~L}$ circular pond, Graesiella sp. WBG-1 showed fast and continued growth $\left(C_{\text {biomass }}\right)$ during 8 days of cultivation under $15 \mathrm{~mol} \mathrm{~m}^{-2}$ day $^{-1}$ artificial light irradiation. Although the nitrate in the algal suspension got depleted from day 2 (Additional file 2), the cells still kept fast growth $\left(C_{\text {biomass }}\right)$ and the growth slightly slowed down from day 5 . The overall biomass productivity $\left(P_{\text {biomass }}\right)$ was $12.03 \mathrm{~g} \mathrm{~m}^{-2}$ day $^{-1}$. From day 2 to day 5 , the biomass productivities maintained at $\sim 14 \mathrm{~g} \mathrm{~m}^{-2}$ day $^{-1}$. The maximum biomass productivity (15.65 $\mathrm{g} \mathrm{m}^{-2}$ day $^{-1}$ ) was achieved on day 2 .

Cultivation of Graesiella sp. WBG-1 in 30 L tank PBR was carried out twice under direct sun light irradiation. The first cultivation (Fig. 2b) was carried out in June 2012 with an average light intensity of $28 \mathrm{~mol}$ photon $\mathrm{m}^{-2}$ day $^{-1}$ and air temperature of $23.2{ }^{\circ} \mathrm{C}$. The second time (Fig. 2c) was carried out in July 2012 with an average light intensity of $31 \mathrm{~mol}$ photon $\mathrm{m}^{-2} \mathrm{day}^{-1}$ and air temperature of $24.8^{\circ} \mathrm{C}$.

For both cultivations, no obvious adaptation phase was observed in the $30 \mathrm{~L}$ tank PBR, and the $C_{\text {biomass }}$ increased continuously with slight fluctuation in growth rate, perhaps, due to fluctuating natural irradiance (Additional file 3). The biomass productivity fluctuated even more through time with fluctuating light. At the end of cultivation, very similar $C_{\text {biomass }}\left(\sim 155 \mathrm{~g} \mathrm{~m}^{-2}\right)$ was achieved in both experiments. The average $P_{\text {biomass }}$ was $17.53 \mathrm{~g} \mathrm{~m}^{-2}$ day $^{-1}$ for the first cultivation (June 2012) and $18.85 \mathrm{~g} \mathrm{~m}^{-2}$ day $^{-1}$ for the second cultivation (July 2012), respectively.

The seed Graesiella sp. WBG-1 cultured under nitrate sufficient condition only contained a small proportion of lipids (18.01\% DW), of which $47.22 \%$ were TAGs. After 8 days of cultivation in the $10 \mathrm{~L}$ circular pond, the $C_{\text {lipid }}$ increased to $45.23 \% \mathrm{DW}$ and over $86 \%$ of the lipids were TAGs (Table 1). Compared to the small-scale cultivation, lower $C_{\text {lipid }}$ was achieved in $30 \mathrm{~L}$ outdoor cultivations, partly because of lower availability of biomass-specific light (Discuss in detail later). However, the lipid productivity $\left(P_{\text {lipid }}\right)$ achieved in outdoor $30 \mathrm{~L}$ cultivations was higher than those of $10 \mathrm{~L}$ cultivations, because more biomass (though containing lower lipid) was produced per illuminated area. 

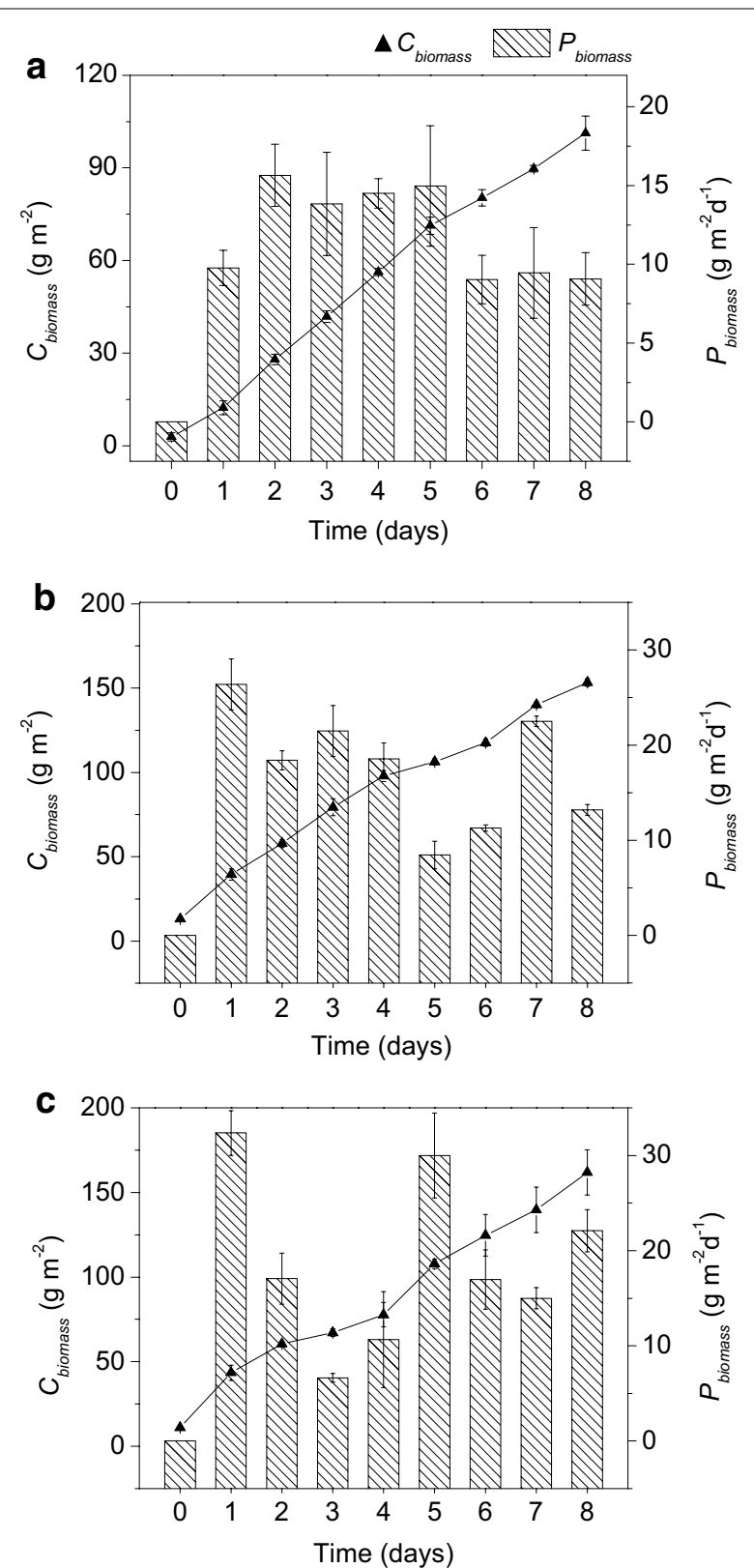

Fig. 2 Microalgal growth and biomass productivity in small-scale reactor. a Cultivation in $10 \mathrm{~L}$ laboratory circular pond; b cultivation in $30 \mathrm{~L}$ outdoor tank PBR in June 2012; and c cultivation in $30 \mathrm{~L}$ outdoor tank PBR in July 2012. Time evolution of biomass concentrations and biomass productivities are indicated by line and column, respectively. The areal biomass concentration $\left(C_{\text {biomass }} \mathrm{g} \mathrm{m}^{-2}\right.$ ) was used in all outdoor experiments

\section{Cultivation of Graesiella sp. WBG-1 in outdoor $200 \mathrm{~m}^{2}$ raceway pond}

Three batch cultures of the Graesiella sp. WBG-1 in a $200 \mathrm{~m}^{2}$ raceway pond were carried out three times in June 2013, July 2013 and May 2014. Figure 3 shows the changes of the culture color as well as the morphologic appearance of the cells. At the early stage of the batch culture, the microalgae suspension looked dark green and a pyrenoid could be observed in the cell. With the aging of culture, the pyrenoid was disappeared and the culture color was yellowish. After 15 days of cultivation, yellow-green or orange color could be seen in the raceway pond.

The time evolution of biomass concentration, lipid productivity, as well as natural light intensity, during the first cultivation in June 2013, were shown in Fig. 4 as a typical example, and a summary $\left(C_{\text {biomass }}, C_{\text {lipid }}, P_{\text {biomass }}, P_{\text {lipid }}\right.$, et al.) of the three cultivations was illustrated in Table 2 .

As shown in the middle panel of Fig. 4, the microalga grew rapidly in the first 4 days of cultivation in the raceway pond, and the $C_{\text {biomass }}$ increased from 7.86 to $65.67 \mathrm{~g} \mathrm{~m}^{-2}$. After day 4, the cell growth slowed down, because the nitrate in the algal suspension was depleted (Additional file 2) and the natural light intensity was reduced (upper panel of Fig. 4).

As shown in the lower panel of Fig. 4, the $C_{\text {lipid }}$ of Graesiella sp. WBG-1 decreased in the first 2 days of cultivation and then increased afterwards, from 14.70 to $31.82 \%$ DW. The $P_{\text {lipid }}$ showed positive correlation with the $P_{\text {biomass }}$, suggesting the essential role of biomass productivity in lipid production. Fluorescence microscopic observation (Fig. 3) also demonstrated the increase in lipid content in Graesiella sp. WBG-1 cells.

The biochemical compositions and fatty acid profiles of Graesiella sp. WBG-1 in $200 \mathrm{~m}^{2}$ raceway cultivation were also examined on the day 4 and day 15 of cultivation, when the cells were under the nitrate starvation condition. The carbohydrate content as a percentage of dry biomass did not show a significant change in all three experiments, while the protein content decreased with aging of the culture (Fig. 5a). A significant increase was observed in total lipids, especially the storage TAGs (Fig. 5a; Table 2), which made up $28.76 \%$ of the dry biomass on the day 15. In contrast, the storage TAGs on the day 4 were only made up $16.67 \%$ of the dry biomass. The fatty acid profile was analyzed using total lipids instead of TAGs, because DAGs and other free fatty acids in the total lipids can also be used to form fatty acid methyl esters. As shown in Fig. 5b, the most abundant fatty acids in Graesiella sp. WBG-1 cultivated in the $200 \mathrm{~m}^{2}$ raceway pond were $\mathrm{C} 16-\mathrm{C} 18$ (especially $\mathrm{C} 16: 0$ and $\mathrm{C} 18: 1$ ), and the amounts of $\mathrm{C} 5-\mathrm{C} 15$ and $\mathrm{C} 1-9 \mathrm{C} 24$ fatty acids were relatively low.

\section{Discussion}

Optimization of nitrate and bicarbonate concentration Nitrogen deficiency is the primary stress to alter algal metabolism to TAG synthesis, while limit cell growth at the same time $[8,19]$. These general trends were also 
Table 1 Lipid production in the two small-scale reactors and corresponding light conditions

\begin{tabular}{lllll}
\hline Trials & $\boldsymbol{I}_{\text {biomass }}\left(\mathbf{m o l ~ g}^{\mathbf{- 1}} \mathbf{D W ~ d a y}{ }^{-\mathbf{1}}\right)$ & $\boldsymbol{C}_{\text {lipid }}(\% \mathrm{DW})$ & TAGs $(\% \mathrm{DW})$ & $\boldsymbol{P}_{\text {lipid }}\left(\mathbf{g ~ m}^{\mathbf{- 2}} \mathbf{d a y}^{\mathbf{- 1}}\right)$ \\
\hline $10 \mathrm{~L}$ & $0.2-5.3$ & $45.2 \pm 1.2$ & $39.3 \pm 0.5$ & $5.7 \pm 0.8$ \\
$30 \mathrm{~L}$ (June 2012) & $0.2-2.1$ & $35.6 \pm 1.0$ & $30.8 \pm 1.0$ & $6.5 \pm 0.8$ \\
$30 \mathrm{~L}$ (July 2012) & $0.2-2.8$ & $32.9 \pm 1.3$ & $30.0 \pm 0.4$ & $6.4 \pm 1.2$ \\
\hline
\end{tabular}

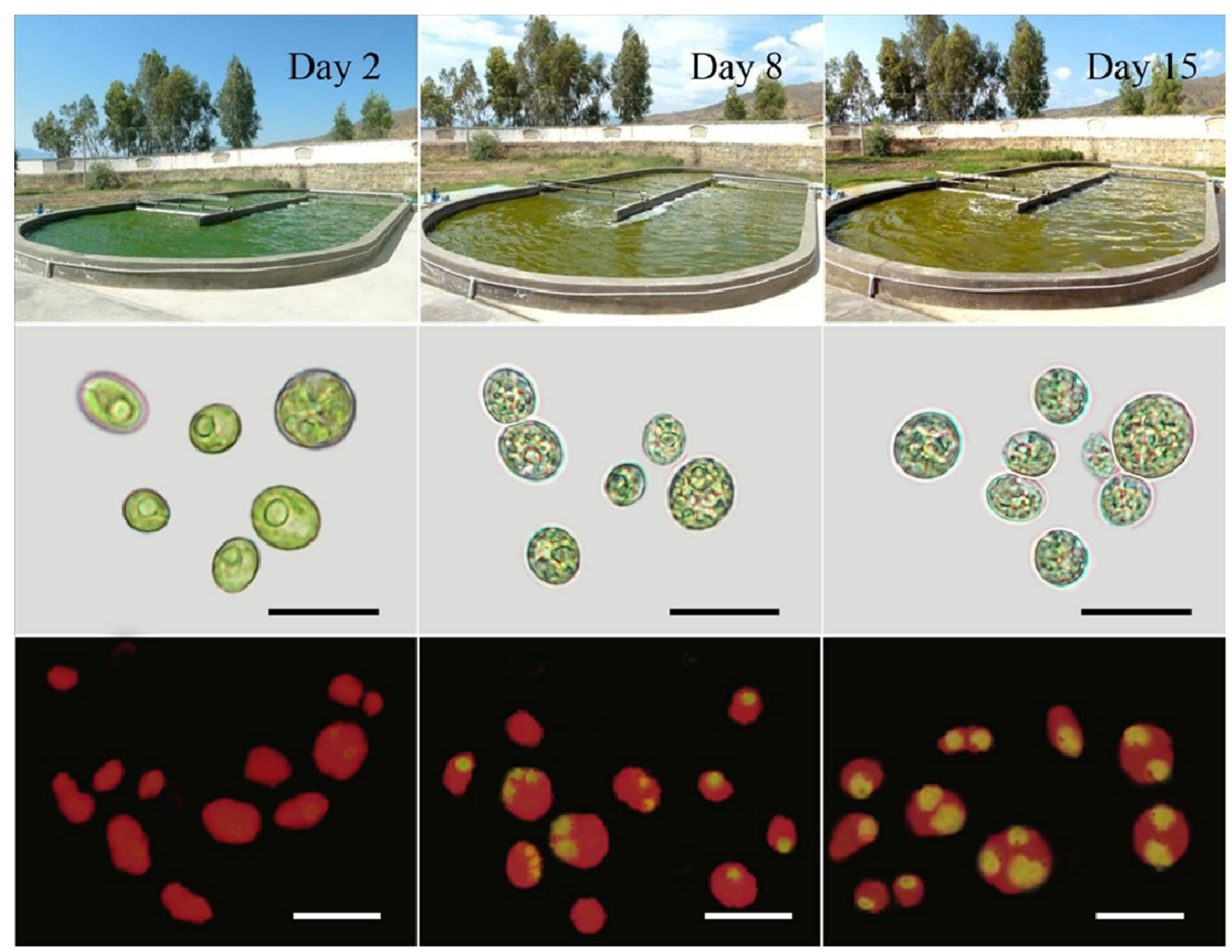

Fig. 3 Cultivation of Graesiella sp. WBG-1 in $200 \mathrm{~m}^{2}$ raceway pond. Changes in culture color and morphology of the cells during cultivation were shown. Intracellular lipid bodies were stained using Nile Red (yellow) and auto-fluorescence of chloroplasts is seen in red. Scale bar $10 \mu \mathrm{m}$

observed in Graesiella sp. WBG-1, as shown in Fig. 1b. Adams et al. [20] pointed out that culturing either many cells with low lipid content or few cells with high lipid content will not result in an economically viable biodiesel feedstock, although both cases give high lipid productivity. In this sense, the initial nitrate concentrations of 0.59 and $5.88 \mathrm{mM}$, in this study (Fig. 1b), will not suitable for microalgal lipid production. The optimal nitrate concentration range for lipid production should be 1.18$3.53 \mathrm{mM}$ in this study (Fig. 1b) to balance the trade-off between the growth and lipid accumulation of Graesiella sp. WBG-1.
On the other side, initial nitrate concentration of a simple bath culture also put strong influence on biomass density. To some extent, the higher the initial nitrate concentration, the higher the biomass density could be achieved (Fig. 1b). However, when biomass density is low, light has good penetration and individual cells are exposed to a large quantity of light energy [18], resulting in more metabolic carbon flux to be channeled to lipid accumulation [21, 22]. Our observation was consistent with these conclusions, showing that low nitrate concentration not only resulted in high $C_{\text {lipid }}$, but also low biomass density (Fig. 1b), which helped to form high light 


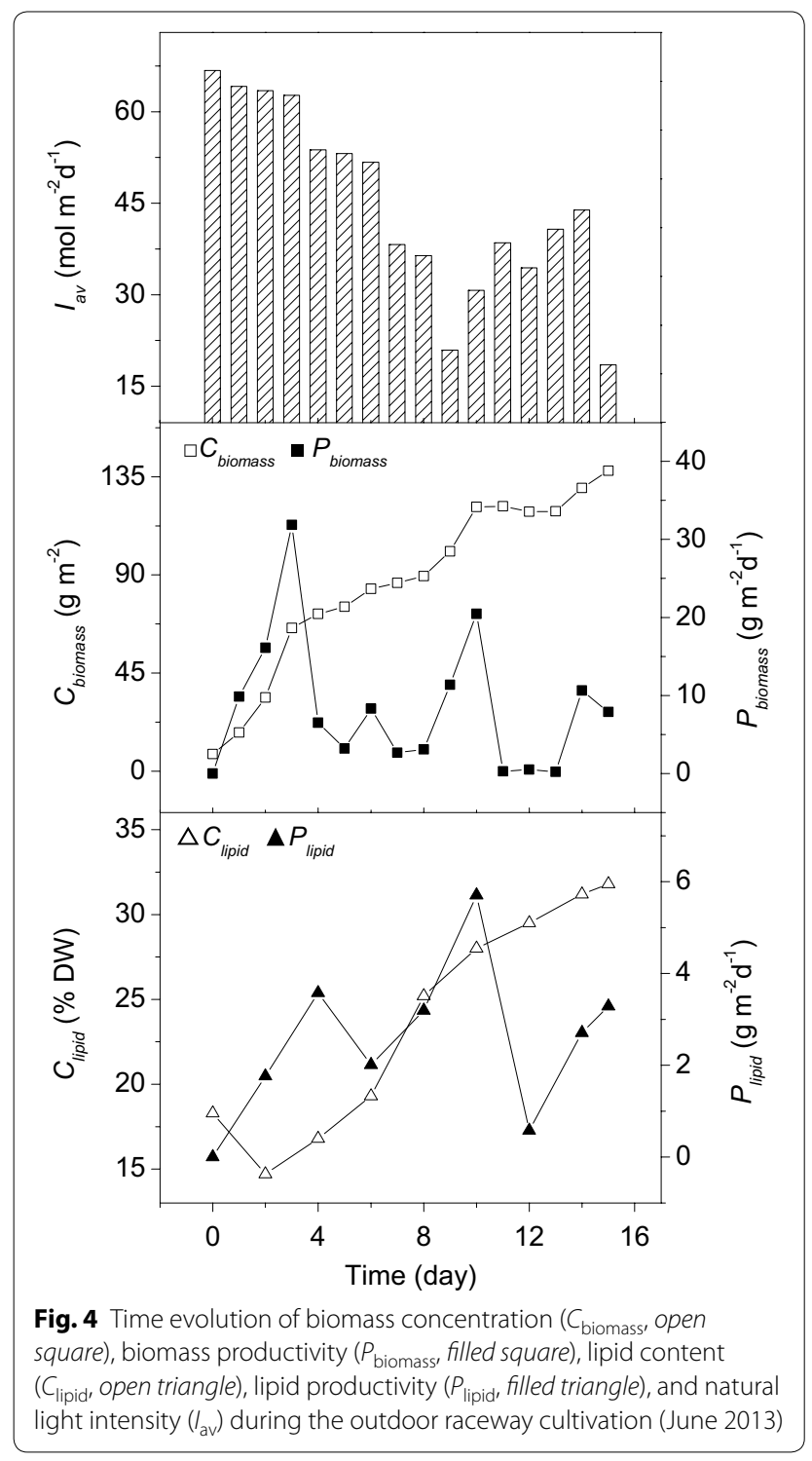

availability $\left(I_{\text {biomass }}\right)$ in the culture (Fig. 6). $I_{\text {biomass }}$ was in turn favorable for high lipid content $\left(C_{\text {lipid }}\right)$ [18]. Therefore, the optimal nitrate concentration should be able to give sufficient light availability per gram of biomass.
$I_{\text {biomass }}$ varies depending on reactor setup as well as light intensity and initial nutrition. A rough simulation revealed a huge difference in $I_{\text {biomass }}$ among four reactors used in this study (Additional file 4). Given the same volumetric biomass concentration, $I_{\text {biomass }}$ in $200 \mathrm{~mL}$ column was four times higher than that in the outdoor $30 \mathrm{~L}$ tank PBR, and $I_{\text {biomass }}$ in $30 \mathrm{~L}$ tank PBR and $200 \mathrm{~m}^{2}$ raceway pond were very similar. Thus, compared to the cultivation in $200 \mathrm{~mL}$ column, a lower biomass concentration $\left(C_{\text {biomass }}\right)$ is needed for $30 \mathrm{~L}$ tank PBR and $200 \mathrm{~m}^{2}$ raceway pond, to give a relatively higher $I_{\text {biomass }}$ to facilitate lipid accumulation. Therefore, among the optimal nitrate concentration range of $1.18-3.53 \mathrm{mM}$ for lipid production, $1.18 \mathrm{mM}$ nitrate is the best concentration for following outdoor cultivation. By sacrificing some of the algal growth using reduced initial nitrate concentration, fairly high light availability $\left(I_{\text {biomass }}\right)$ could be achieved.

Several studies have demonstrated the importance of photosynthesis efficiency on oil formation [18, 21, 23]. In other words, normal photosynthesis is needed to produce enough carbon flux and drive the biosynthesis of TAGs under nitrogen stress. Actually, carbon in cultures with air-blowing or paddle wheel stirring is far from sufficient $[24,25]$. Thus, addition of carbon is another possible way to improve microalgal lipid production. White et al. [26] reported that addition of bicarbonate could significantly increase cell abundance in culture of Tetraselmis suecica and Nannochloropsis salina. We got a similar result using Graesiella sp. WBG-1 (Fig. 1a), although the $C_{\text {biomass }}$ were statistically insignificant between 11.90 and $23.81 \mathrm{mM}$ bicarbonate. Lipid accumulation, however, was unaffected by different bicarbonate concentrations in this study. The optimal bicarbonate concentration for Graesiella sp. WBG-1 cultivation, therefore, is $11.90 \mathrm{mM}$ considering the culture cost.

Performance of Graesiella sp. WBG-1 in scale-up cultivation The $10 \mathrm{~L}$ photobioreactor used in this study is a minitype industrial circular pond, equipped with light, temperature, and $\mathrm{pH}$ control systems. In this reactor, the strain Graesiella sp. WBG-1 showed good growth

Table 2 Summary of the cultivations at different scale

\begin{tabular}{|c|c|c|c|c|c|c|c|}
\hline Cultures & $\begin{array}{l}\text { Duration } \\
\text { (days) }\end{array}$ & $\begin{array}{l}l_{\mathrm{av}} \\
\left(\mathrm{mol} \mathrm{m}^{-2} \mathrm{day}^{-1}\right)\end{array}$ & $\begin{array}{l}I_{\text {biomass }} \\
\left(\mathrm{mol} \mathrm{g}^{-1} \mathrm{DW} \mathrm{day}^{-1}\right)\end{array}$ & $\begin{array}{l}C_{\text {biomass }} \\
\left(\mathrm{g} \mathrm{m}^{-2}\right)\end{array}$ & $\begin{array}{l}C_{\text {lipid }} \\
(\% \text { DW })\end{array}$ & $\begin{array}{l}P_{\text {biomass }} \\
\left(\mathrm{g} \mathrm{m}^{-2} \text { day }^{-1}\right)\end{array}$ & $\begin{array}{l}P_{\text {lipid }} \\
\left(\mathrm{g} \mathrm{m}^{-2} \text { day }^{-1}\right)\end{array}$ \\
\hline $10 \mathrm{~L}$ circular pond & 8 & 15 & $0.2-5.38$ & 101.2 & 45.2 & 12.0 & 5.7 \\
\hline 30 L tank (June 2012) & 8 & 33 & $0.2-2.1$ & 153.3 & 35.6 & 17.5 & 6.5 \\
\hline 30 L tank (July 2012) & 8 & 29 & $0.2-2.8$ & 161.8 & 32.9 & 18.9 & 6.4 \\
\hline Raceway (June 2013) & 15 & 45 & $03-5.7$ & 137.8 & 31.8 & 8.7 & 2.9 \\
\hline Raceway (July 2013) & 13 & 36 & $0.3-4.4$ & 102.3 & 33.4 & 7.2 & 2.4 \\
\hline Raceway (May 2014) & 14 & 39 & $0.4-3.4$ & 97.7 & 29.4 & 6.2 & 2.0 \\
\hline
\end{tabular}



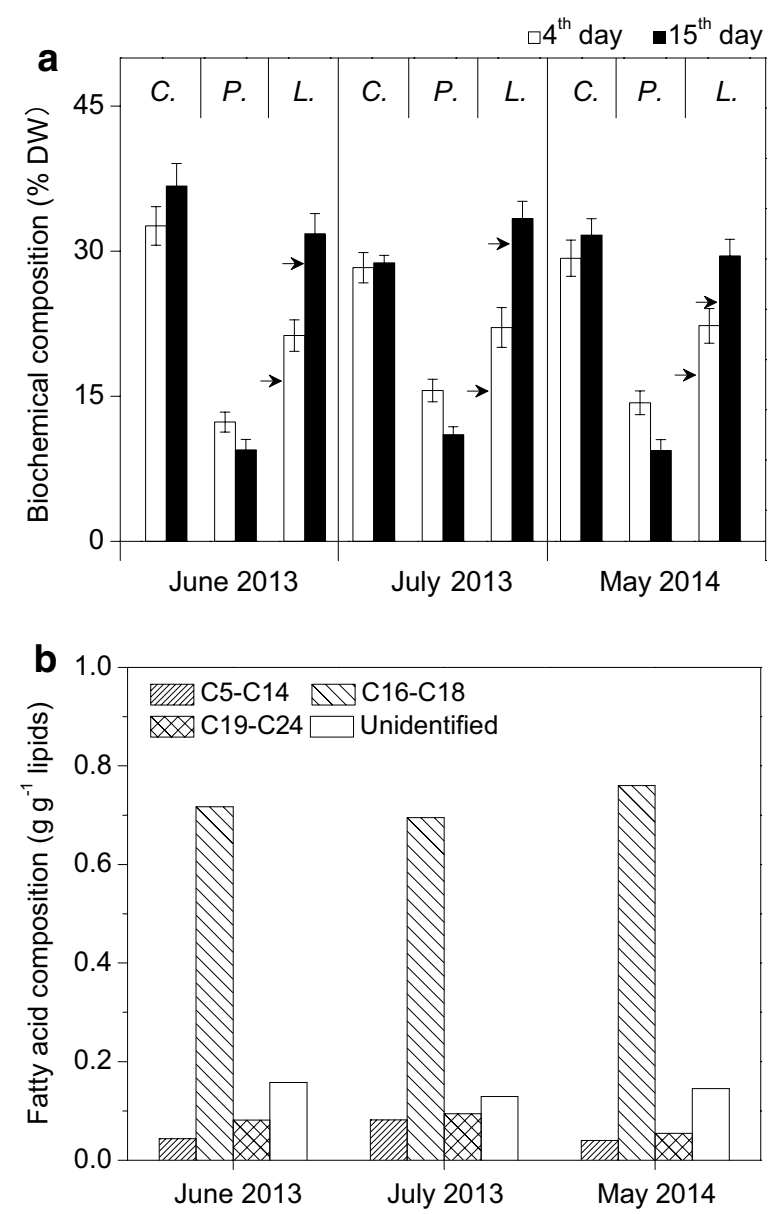

Fig. 5 Biochemical compositions (a) and fatty acid profiles (b) of Graesiella sp. WBG-1 in $200 \mathrm{~m}^{2}$ raceway cultivation. Carbohydrate, protein, and lipid at the 4th day (open column) and 15th day (filled column) are denoted with $C_{\text {., }}$., and L., respectively. Arrows indicate TAGs content on dry biomass bases (\% DW)

(12.03 $\mathrm{g} \mathrm{m}^{-2}$ day $\left.^{-1} P_{\text {biomass }}\right)$ and high $C_{\text {lipid }}(45.23 \% \mathrm{DW})$ (Table 2), consistent with the previous results observed in our laboratory. Compared to the $10 \mathrm{~L}$ photobioreactor, higher $P_{\text {biomass }}\left(17.53-18.85 \mathrm{~g} \mathrm{~m}^{-2}\right.$ day $\left.^{-1}\right)$ was achieved in outdoor cultivation in $30 \mathrm{~L}$ tank PBR. However, the $C_{\text {lipid }}(32.89-35.63 \% \mathrm{DW})$ decreased in $30 \mathrm{~L}$ tank PBR. The decrease in lipid content could be partly interpreted as a result of lower availability of biomass-specific light ( $I_{\text {biomass, }}$, the average light irradiance received by each gram of biomass) [18]. Light is an important stress factor for microalgal lipid accumulation [8]. It is also reported that the de novo synthesis of intracellular TAGs is highly correlated with photosynthetic activity as well as light energy available for microalgal cells [21, 23]. In this sense, sufficient light supply (higher $I_{\text {biomass }}$ ) is helpful to get a high lipid content.

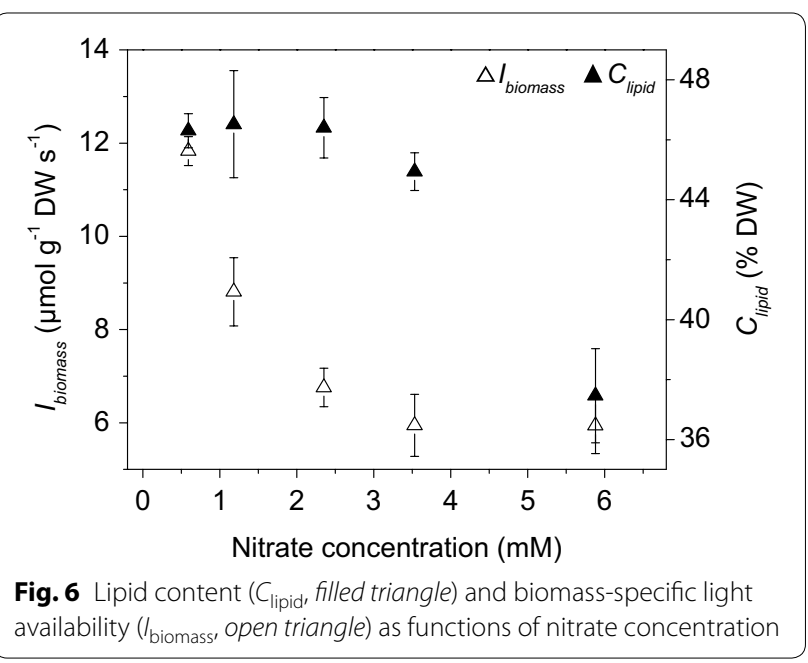

In fact, the $30 \mathrm{~L}$ tank PBR has a longer light path $(15 \mathrm{~cm})$ than the $10 \mathrm{~L}$ circular pond $(10 \mathrm{~cm})$, giving a much lower biomass-specific light availability $\left(I_{\text {biomass }}\right)$. Although lower $C_{\text {lipid }}$ was achieved in $30 \mathrm{~L}$ tank PBR, $P_{\text {biomass }}$ was significantly higher than achieved in $10 \mathrm{~L}$ circular pond, because there were more growing cells per illuminated area in the $30 \mathrm{~L}$ tank PBR. As a result, higher lipid productivity was achieved in the $30 \mathrm{~L}$ tank PBR. On the other hand, outdoor light irradiance was fluctuated in a wide range of $16-44 \mathrm{~mol} \mathrm{~m}^{-2}$ day $^{-1}$ during the experiments (Additional file 3), and the highest instant light irradiance reached $2500 \mu \mathrm{mol} \mathrm{m} \mathrm{m}^{-2} \mathrm{~s}^{-1}$, but no lag phase was observed in both laboratory and outdoor conditions. These results indicated the nice adaptability of Graesiella sp. WBG-1 to strong and fluctuating natural solar irradiance.

Cultivation of Graesiella sp. WBG-1 in $200 \mathrm{~m}^{2}$ raceway pond was successfully carried out three times in the summer at Chenghai town, where most days are sunny throughout the spring and early summer. The average daily light intensity during these three cultivations was 45,36 , and $39 \mathrm{~mol} \mathrm{~m}^{-2}$ day $^{-1}$, respectively, which was two-to-three times as high as the artificial light intensity in the $10 \mathrm{~L}$ circular pond. Under such light irradiance, the daily average $P_{\text {biomass }}$ achieved in $200 \mathrm{~m}^{2}$ raceway pond was $6.17-8.66 \mathrm{~g} \mathrm{~m}^{-2} \mathrm{day}^{-1}$, substantially lower than the productivity previously reported in studies of microalgal mass production in food/feed industry [27]. Nevertheless, a maximum $P_{\text {biomass }}$ of $31.86 \mathrm{~g} \mathrm{~m}^{-2}$ day $^{-1}$ in $200 \mathrm{~m}^{2}$ raceway pond (Fig. 4) was reached in the first 3 days when nitrate was sufficient. The marked difference between average $P_{\text {biomass }}$ and maximum $P_{\text {biomass }}$ suggested that the cell growth was severely limited by nitrate depletion in the later 12 days of cultivation. Actually, the initial nitrate 
concentration was only $1.18 \mathrm{mM}$, which was insufficient for the alga to grow 15 days in $200 \mathrm{~m}^{2}$ raceway pond. On the other hand, lower initial nitrate concentration only supported lower $C_{\text {biomass }}$, and thus resulted in higher $I_{\text {bio- }}$ mass, which was found to promote lipid accumulation [18]. As a result, relatively high $C_{\text {lipid }}(29.41-33.42 \% \mathrm{DW})$ was achieved in the $200 \mathrm{~m}^{2}$ raceway pond. If the initial nitrate concentration was increased from 1.18 to $1.76 \mathrm{mM}$, no increase in the $C_{\text {lipid }}$ of Graesiella sp. WBG-1 could be observed after 15 days of cultivation in raceway pond (unpublished data). Therefore, the biological processes of cell growth and lipid accumulation should be further studied to optimize momentum, energy, and mass transfer in raceway pond.

Although the lipid content of $29.41-33.42 \%$ DW is much lower than achieved in the small-scale cultivation (nitrate-deplete) in laboratory, it is of great importance for two reasons. First, Chlorophyta strains are reported to have a $C_{\text {lipid }}$ of $10-25 \% \mathrm{DW}$ under nitrate-replete conditions [28]. For the microalga Graesiella sp. WBG1 , the $C_{\text {lipid }}$ is about $14-17 \% \mathrm{DW}$ under nitrate-replete conditions (Fig. 4). The final $C_{\text {lipid }}$ of 29.41-33.42\% DW obtained in the $200 \mathrm{~m}^{2}$ raceway pond under nitratedepleted conditions is nearly two times the initial value. Furthermore, TAGs contributed a large proportion $(84.38-91.88 \%)$ of the total lipids in the harvested cell mass, and $\mathrm{C} 16-\mathrm{C} 18$ were the major fatty acids, comprising more than $70 \%$ of total fatty acids. These data clearly indicated that lipid was markedly accumulated in the cells of Graesiella sp. WBG-1 cultured in the $200 \mathrm{~m}^{2}$ raceway pond.

Second, sharp declines in $C_{\text {lipid }}$ with increasing culture volumes have been observed in many studies. For example, Li et al. [10] reported that Parachlorella kessleri CCALA 255 had a lipid content of $55 \%$ DW in laboratory, but only $25 \%$ DW when cultured in a Large-Scale Industrial Thin-Layer Bioreactor. In a $2000 \mathrm{~L}$ raceway pond, the green microalga Botryococcus bruanii AP103 reached a total lipid content of only $30.8 \%$ DW, after 15 days of outdoor cultivation [11]. More recently, more than $50 \%$ decrease in lipid content (from 40 to $19.8 \%$ DW) was observed in Chlorella strain $\mathrm{CH} 2$, when its cultivation was scaled up from 0.25 GWP to 1 ha GWP [12]. In other studies in our lab, although some strains of Chlorella and Scenedesmus can accumulate large amounts of lipids (over $50 \%$ DW) in laboratory, their lipid content are similar to the initial values $(\sim 20 \% \mathrm{DW})$, and there is almost no net increase in TAGs when cultivated in the raceway pond (Additional file 5) with the same technology described in this study. However, the decline in $C_{\text {lipid }}$ of the microalga Graesiella sp. WBG-1 cultured in the $200 \mathrm{~m}^{2}$ raceway pond is less than the other tested microalgae. Moreover, more than one-fold increase in
TAGs was observed. These phenomena suggest that lipid accumulation does not always occur for any oleaginous microalgae in mass open culture. One possible explanation is the loss of culture competitiveness [13]. This is why the field test is necessary for oleaginous microalgae selection. As discussed above, Graesiella sp. WBG-1 is a potential oil producer with wide tolerance to natural environmental conditions.

\section{Other features of Graesiella sp. WBG-1 for large-scale cultivation in open ponds}

As indicated in many studies, lipid productivity is not the sole criterion for the selection of lipid-rich microalgae. One of the desirable characteristics for mass culture is large cell size [28], which is assumed to be easier to sediment in liquid culture [29] and reduce harvesting and down-stream processing costs. Graesiella sp. WBG-1 cells are broadly ellipsoidal or globose with a diameter of $8-12 \mu \mathrm{m}$, which is significantly larger than other reported oleaginous microalgae, such as Chlorella (diameter 3-8 $\mu \mathrm{m}$ ). It was observed in this study that Graesiella sp. WBG-1 cells settle down easily in medium in a short time, and this feature becomes more obvious with the aging of culture. The high culture $\mathrm{pH}(9.0 \pm 0.5$ in this study) may also play a role in the auto-participation [30]. Over $98 \%$ of the cells settled down to the bottom of the raceway pond when the algal suspension was kept without stirring over night. By removing the upper layer medium from the pond, the algal biomass was concentrated more than 50 times (Fig. 7). This feature potentially reduce liquid volume for harvesting if a standing procedure is employed before centrifugation or filtration, and the harvesting cost is reduced then.

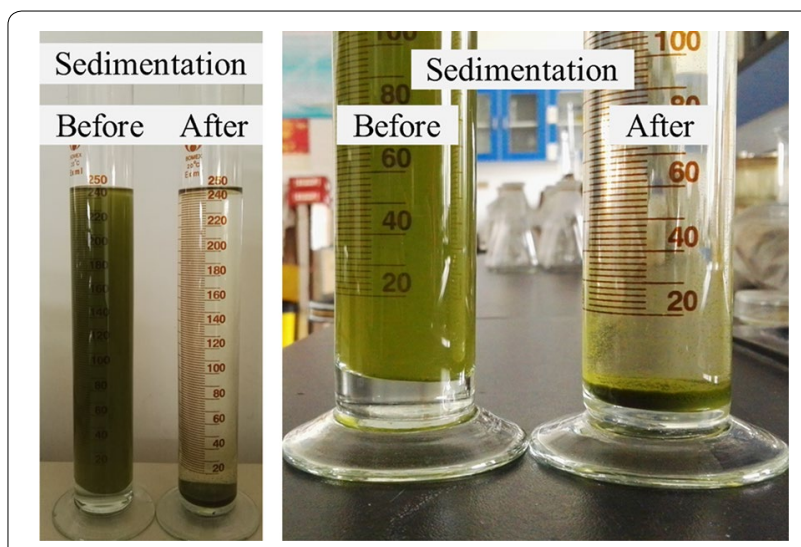

Fig. 7 Sedimentation of Graesiella sp. WBG-1 cells over night. The homogeneous cell suspension (left) was kept still in a glass cylinder and over $98 \%$ of biomass sedimented to the bottom after about $8 \mathrm{~h}$ (right) 
Biological contamination is a serious problem for microalgae cultivation in open pond [31]. Protozoa may be predators on algae, bacteria and fungi, may be parasites, and other non-target microalgae may compete with the cultured algae for light and nutrients [32]. Theoretically, the ability to resist contamination is different among different algal strains, because there are many factors that can potentially inhibit the growth of contaminated organism, such as microalgal cell size and their tolerance to extreme environmental conditions, including temperature, $\mathrm{pH}$, and salinity. Recently polysaccharides from algae are reported to have antibacterial activity [33], suggesting allelopathy is a prospective way for contamination control in microalgae mass cultivation [34]. In our experience, Graesiella sp. WBG-1 is more resistant to contamination compared to other tested algae. Although invading organisms, such as bacteria and zooplankton, could be identified under microscope in Graesiella sp. WBG-1 culture, but the number of these organisms was limited and the negative effects on productivity was negligible. In contrast, zooplankton contamination often led to culture collapse in the open cultivation of Chlorella, Scenedesmus, Desmodesmus, and Chlorococcum. It is not clear if Graesiella sp. WBG-1 has some specific chemical defense system or not, but Graesiella sp. WBG-1 is an indigenous strain isolated from Chenghai Lake and well adapted to the environment. Maybe, its large cell size can cause swallowing difficulty for protozoa.

Coupling bio-fixation of carbon dioxide with microalgae triacylglycerides production is a hot research topic worldwide [35, 36]. It is known that sufficient gas-liquid contact is important for efficient absorption of $\mathrm{CO}_{2}$ [36], and high $\mathrm{pH}$ can prevent the escape of $\mathrm{CO}_{2}$ from culture [37]. In this study, a microporous tubular gas diffuser (average pore size $<100 \mu \mathrm{m}$ ) was used to maximize $\mathrm{CO}_{2}$ water contact, and a high culture $\mathrm{pH}(9.0 \pm 0.5)$, which had no negative effects on Graesiella sp. WBG-1 growth and lipid accumulation (unpublished data), was maintained in $200 \mathrm{~m}^{2}$ raceway to prevent the escape of $\mathrm{CO}_{2}$. Under such condition, a $\mathrm{CO}_{2}$ utilization rate (bio-carbon divide by consumed carbon) of $65.7 \%$ was achieved. This utilization rate is even higher than that those achieved in the mass culture of Spirulina (60\%) [38, 39], Chlorella (46\%) [40], and Phaeodactylum (63\%) [41]. These results indicate that Graesiella sp. WBG-1 can readily be used for microalgal mass production coupled with $\mathrm{CO}_{2}$ fixation.

\section{Conclusions}

This study identified a lipid-rich microalgal strain Graesiella sp. WBG-1. We assessed its growth and lipid production under laboratory and outdoor conditions, and demonstrated that Graesiella sp. WBG-1 is an industrial strain capable of high lipid productivity in outdoor mass cultivation. The results can greatly enrich our knowledge of the behavior of oleaginous microalga in outdoor open raceway pond. To the best of our knowledge, this is one of the few reports on integrated indoor and outdoor cultivation for oleaginous microalgae selection. A high lipid content of $33.4 \%$ DW was achieved for the first time in a $200 \mathrm{~m}^{2}$ raceway pond. The results also provide evidence for the feasibility of using low-cost raceway pond for autotrophic cultivation of microalgae for lipid production.

\section{Additional files}

Additional file 1. Preliminary selection of the 63 microalgae strains in a bubbled column photobioreactor.

Additional file 2. Changes of residual nitrate concentration during the experiments.

Additional file 3. Daily light irradiance and air temperature during the outdoor trials.

Additional file 4. Simulation of biomass-specific light availability in the used reactors based on different volumetric biomass concentration.

Additional file 5. Biomass concentration and lipid content of several other microalgae cultured in different PBRs.

\section{Abbreviations}

PBR: photobioreactor; DW: dry weight; $C_{\text {biomass: }}$ dry biomass concentration; $C_{\text {lipid }}$ : lipid content; $P_{\text {biomass: }}$ : biomass productivity; $P_{\text {lipid: }}$ : lipid productivity; $I_{\text {av: }}$ : average light intensity at the PBR surface or culture suspension surface; I biomass: biomass-specific light availability; $R_{c}: \mathrm{CO}_{2}$ utilization rate.

\section{Authors' contributions}

XW conceived, designed the experiments, performed the laboratory work, analyzed, and interpreted the data and drafted the paper. KD and ZW performed the outdoor cultivations and revised the manuscript. XP, HT, and $Y X$ analyzed the biomass lipid, protein, and carbohydrate, and performed the statistical analysis. $\mathrm{LL}$ designed the measurement of $\mathrm{CO}_{2}$ utilization rate, interpreted the data. and critically revised the manuscript. DZ and YG maintained the seed culture and contributed to the scale-up cultivation. YL contributed to experimental design and data interpretation and critically revised the manuscript. All authors read and approved the final manuscript.

\section{Author details \\ ${ }^{1}$ Key Laboratory of Pant Germplasm Enhancement and Specialty Agriculture, Wuhan Botanical Garden, Chinese Academy of Sciences, Wuhan 430074, China. ${ }^{2}$ University of Chinese Academy of Sciences, Beijing 100049, China. \\ ${ }^{3}$ Present Address: Department of Pathology and Immunology, Baylor College of Medicine, Houston, TX 78703, USA.}

\section{Acknowledgements}

The authors acknowledge the financial support for this work from the Ministry of Science and Technology of China (Nos. 2012FY112900, 2013AA065805) and China Petrochemical Corporation (Nos. 210080, 215104).

\section{Availability of supporting data}

The data sets supporting the results of this article are included within the article and its additional files.

\section{Competing interests}

The authors declare that they have no competing interests. 


\section{Consent for publication}

All authors have approved the manuscript to be published.

\section{Funding}

This work is jointly financed by the Ministry of Science and Technology of China (Nos. 2012FY112900, 2013AA065805), and China Petrochemical Corporation (Nos. 210080, 215104).

Received: 29 February 2016 Accepted: 2 June 2016 Published online: 13 June 2016

\section{References}

1. Hu Q. Industrial production of microalgal cell mass and secondary products-major industrial species. In: Richmond A, editor. Handbook of microalgal culture: biotechnology and applied phycology. Oxford: Wiley; 2004. p. 264-72.

2. Pulz O, Gross W. Valuable products from biotechnology of microalgae. Appl Microbiol Biotechnol. 2004;65:635-48.

3. Singh B, Guldhe A, Rawat I, Bux F. Towards a sustainable approach for development of biodiesel from plant and microalgae. Renew Sustain Energy Rev. 2014;29:216-45.

4. Wobbe L, Remacle C. Improving the sunlight-to-biomass conversion efficiency in microalgal biofactories. J Biotechnol. 2014;201:28-42.

5. Klok AJ, Martens DE, Wijffels RH, Lamers PP. Simultaneous growth and neutral lipid accumulation in microalgae. Bioresour Technol. 2013;134:233-43.

6. Wijffels RH, Barbosa MJ. An outlook on microalgal biofuels. Science. 2010;329:796-9.

7. Stephens E, Ross IL, Mussgnug JH, Wagner LD, Borowitzka MA, Posten C, Kruse O, Hankamer B. Future prospects of microalgal biofuel production systems. Trends Plant Sci. 2010;15:554-64.

8. Hu Q, Sommerfeld M, Jarvis E, Ghirardi M, Posewitz M, Seibert M, Darzins A. Microalgal triacylglycerols as feedstocks for biofuel production: perspectives and advances. Plant J. 2008;54:621-39.

9. Gouveia L, Oliveira AC. Microalgae as a raw material for biofuels production. J Ind Microbiol Biotechnol. 2009;36:269-74.

10. Li XL, Pribyl P, Bisova K, Kawano S, Cepak V, Zachleder V, Cizkova M, Branyikova I, Vitova M. The microalga Parachlorella kessleri-a novel highly efficient lipid producer. Biotechnol Bioeng. 2013;110:97-107.

11. Ashokkumar $\bigvee$, Rengasamy R. Mass culture of Botryococcus braunii Kutz. under open raceway pond for biofuel production. Bioresour Technol. 2012:104:394-9.

12. Guccione A, Biondi N, Sampietro G, Rodolfi L, Bassi N, Tredici M. Chlorella for protein and biofuels: from strain selection to outdoor cultivation in a Green Wall Panel photobioreactor. Biotechnol Biofuels. 2014;7:84.

13. Sheehan J, Dunnahay T, Benemann J, Roessler P. A look back at the US Department of Energy's Aquatic Species Program: biodiesel from algae. Golden: National Renewable Energy Laboratory. 1998; Report No. NREL/ TP-580-24190.

14. Wen XB, Liang F, Geng YH, Li YG. Two-stage characteristics of lipid production in batch culture of two green microalgae. Fresen Environ Bull. 2014;23:2253-8

15. Collos Y, Mornet F, Sciandra A, Waser N, Larson A, Harrison PJ. An optical method for the rapid measurement of micromolar concentrations of nitrate in marine phytoplankton cultures. J Appl Phycol. 1999;11:179-84.

16. Wen XB, Geng YH, Li YG. Enhanced lipid production in Chlorella pyrenoidosa by continuous culture. Bioresour Technol. 2014;161:297-303.

17. Slocombe SP, Ross M, Thomas N, McNeill S, Stanley MS. A rapid and general method for measurement of protein in microalgal biomass. Bioresour Technol. 2013:129:51-7.

18. Benvenuti G, Bosma R, Klok AJ, Ji F, Lamers PP, Barbosa MJ, Wijffels RH. Microalgal triacylglycerides production in outdoor batch-operated tubular PBRs. Biotechnol Biofuels. 2015;8:100

19. Takagi M, Watanabe $K$, Yamaberi K, Yoshida T. Limited feeding of potassium nitrate for intracellular lipid and triglyceride accumulation of Nannochloris sp. UTEX LB1999. Appl Microbiol Biot. 2000:54:112-7.
20. Adams C, Godfrey V, Wahlen B, Seefeldt L, Bugbee B. Understanding precision nitrogen stress to optimize the growth and lipid content tradeoff in oleaginous green microalgae. Bioresour Technol. 2013;131:188-94.

21. Dillschneider R, Steinweg C, Rosello-Sastre R, Posten C. Biofuels from microalgae: photoconversion efficiency during lipid accumulation. Bioresour Technol. 2013;142:647-54.

22. Courchesne N, Parisien A, Wang B, Lan C. Enhancement of lipid production using biochemical, genetic and transcription factor engineering approaches. J Biotechnol. 2009;141:31-41.

23. Pan YY, Wang ST, Chuang LT, Chang YW, Chen CNN. Isolation of thermotolerant and high lipid content green microalgae: oil accumulation is predominantly controlled by photosystem efficiency during stress treatments in Desmodesmus. Bioresour Technol. 2011;102:10510-7.

24. Su Z, Kang R, Shi S, Cong W, Cai Z. An economical device for carbon supplement in large scale microalgae production. Bioprocess Biosyst Eng. 2008:31:641-5.

25. Berger R, Libby WF. Equilibration of atmospheric carbon dioxide with sea water: possible enzymatic control of the rate. Science. 1969;164:1395-7.

26. White DA, Pagarette A, Rooks P, Ali ST. The effect of sodium bicarbonate supplementation on growth and biochemical composition of marine microalgae cultures. J Appl Phycol. 2012;25:153-65.

27. Tredici MR. Mass production of microalgae: photobioreactors. In: Richmond A, editor. Handbook of microalgal culture: biotechnology and applied phycology. Oxford:Wiley; 2004. p. 178-214.

28. Griffiths MJ, Harrison STL. Lipid productivity as a key characteristic for choosing algal species for biodiesel production. J Appl Phycol. 2009:21:493-507.

29. Chatsungnoen T, Chisti Y. Harvesting microalgae by flocculation-sedimentation. Algal Res. 2016;13:271-83.

30. Wu ZC, Zhu Y, Huang WY, Zhang CW, Li T, Zhang YM, Li AF. Evaluation of flocculation induced by $\mathrm{pH}$ increase for harvesting microalgae and reuse of flocculated medium. Bioresour Technol. 2012;110:496-502.

31. McBride RC, Lopez S, Meenach C, Burnett M, Lee PA, Nohilly F, Behnke C. Contamination management in low cost open algae ponds for biofuels production. Ind Biotechnol. 2014;10:221-7.

32. Wang H, Zhang W, Chen L, Wang J, Liu T. The contamination and control of biological pollutants in mass cultivation of microalgae. Bioresour Technol. 2013;128:745-50.

33. de Jesus Raposo MF, de Morais AMB, de Morais RMSC. Marine polysaccharides from algae with potential biomedical applications. Mar Drugs. 2015;13:2967-3028

34. Bacellar Mendes LB, Vermelho AB. Allelopathy as a potential strategy to improve microalgae cultivation. Biotechnol Biofuels. 2013;6:1-14.

35. Gutierrez-Arriaga CG, Serna-Gonzalez M, Ponce-Ortega JM, El-Halwagi MM. Sustainable integration of algal biodiesel production with steam electric power plants for greenhouse gas mitigation. ACS Sustain Chem Eng. 2014;2:1388-403

36. Kumar A, Ergas S, Yuan X, Sahu A, Zhang QO, Dewulf J, Malcata FX, van Langenhove $\mathrm{H}$. Enhanced $\mathrm{CO}_{2}$ fixation and biofuel production via microalgae: recent developments and future directions. Trends Biotechnol. 2010:28:371-80.

37. Zhang D, Zhu XY, Wen XB, Geng YH, Li YG. Studies on equilibrium pH values of microalgal medium. Acta Hydrobiol Sin. 2014;3:401-6 (in Chinese with english abstract).

38. Li YG, Hu HJ, Gong XM. Studies on the mechanism of $\mathrm{pH}$ value change and carbon conversion ratio of Spirulina media. Chin J Biotechnol. 1996;12:242-8 (in Chinese with english abstract)

39. Belay A. Mass culture of Spirulina outdoors - the earthrise farms experience. In: Vonshak A, editor. Spirulina platensis (Arthrospira): physiology, cell biology and biotechnology. London: Taylor and Francis; 1997. p. 131-58.

40. Ramanan R, Kannan K, Deshkar A, Yadav R, Chakrabarti T. Enhanced algal $\mathrm{CO}_{2}$ sequestration through calcite deposition by Chlorella sp. and Spirulina platensis in a mini-raceway pond. Bioresour Technol. 2009:101:2616-22.

41. SobczukTM, Camacho FG, Rubio FC, Fernandez FGA, Grima EM. Carbon dioxide uptake efficiency by outdoor microalgal cultures in tubular airlift photobioreactors. Biotechnol Bioeng. 2000;67:465-75. 